University of New Hampshire

University of New Hampshire Scholars' Repository

Space Science Center

Institute for the Study of Earth, Oceans, and

Space (EOS)

2000

\title{
Minute-of-Arc Resolution Gamma ray Imaging Experiment-MARGIE
}

\author{
S C. Kappadath \\ Louisiana State University - Baton Rouge \\ P P. Altice \\ Louisiana State University - Baton Rouge \\ M L. Cherry \\ Louisiana State University - Baton Rouge \\ T G. Guzik \\ Louisiana State University - Baton Rouge \\ J G. Stacy \\ Louisiana State University - Baton Rouge
}

See next page for additional authors

Follow this and additional works at: https://scholars.unh.edu/ssc

Part of the Astrophysics and Astronomy Commons

\section{Recommended Citation \\ Minute-of-Arc Resolution Gamma ray Imaging Experiment-MARGIE Kappadath, S. C. and Altice, P. P. and Cherry, M. L. and Guzik, T. G. and Stacy, J. G. and Macri, J. and McConnell, M. L. and Ryan, J. M. and Band, D. L. and Matteson, J. L. and O'Neill, T. J. and Zych, A. D. and Buckley, J. and Hink, P. L., AIP Conference Proceedings, 510, 779-783 (2000), DOI:http://dx.doi.org/10.1063/1.1303304}

This Conference Proceeding is brought to you for free and open access by the Institute for the Study of Earth, Oceans, and Space (EOS) at University of New Hampshire Scholars' Repository. It has been accepted for inclusion in Space Science Center by an authorized administrator of University of New Hampshire Scholars' Repository. For more information, please contact Scholarly.Communication@unh.edu. 


\section{Authors}

S C. Kappadath, P P. Altice, M L. Cherry, T G. Guzik, J G. Stacy, John R. Macri, Mark L. McConnell, James M. Ryan, D L. Band, J L. Matteson, Terence J. O'Neill, Allen Zych, J Buckley, and P L. Hink 


\section{AIP $\mid$ Proceedings}

\section{Minute-of-Arc Resolution Gamma ray Imaging Experiment-MARGIE}

S. C. Kappadath, P. P. Altice, M. L. Cherry, T. G. Guzik, J. G. Stacy, J. Macri, M. L. McConnell, J. M. Ryan, D. L. Band, J. L. Matteson, T. J. O'Neill, A. D. Zych, J. Buckley, and P. L. Hink

Citation: AIP Conference Proceedings 510, 779 (2000); doi: 10.1063/1.1303304

View online: http://dx.doi.org/10.1063/1.1303304

View Table of Contents: http://scitation.aip.org/content/aip/proceeding/aipcp/510?ver=pdfcov

Published by the AIP Publishing

\section{Articles you may be interested in}

A search for gamma-ray burst optical emission with the automated patrol telescope

AIP Conf. Proc. 428, 909 (1998); 10.1063/1.55461

Optical imaging of gamma-ray bursts with the LONEOS telescope

AIP Conf. Proc. 428, 869 (1998); 10.1063/1.55459

Tracking and imaging gamma ray experiment (TIGRE) for 1 to 100 MEV gamma ray astronomy AIP Conf. Proc. 304, 701 (1994); 10.1063/1.45559

Imaging germanium telescope array for gammarays (IGETAGRAY)

AIP Conf. Proc. 211, 224 (1990); 10.1063/1.39687

A gammaray imaging telescope based on liquid xenon

AIP Conf. Proc. 211, 189 (1990); 10.1063/1.39682 


\title{
Minute-of-Arc Resolution Gamma Ray Imaging Experiment - MARGIE
}

\author{
S.C. Kappadath*, P.P. Altice*, M.L. Cherry*, T.G. Guzik*, \\ J.G. Stacy ${ }^{*}$, J. Macri ${ }^{\dagger}$, M.L. McConnell ${ }^{\dagger}$, J.M. Ryan ${ }^{\dagger}$, D.L. Band ${ }^{\S,}$, \\ J.L. Matteson ${ }^{\S}$, T.J. O’Neill!, A.D. Zych", J. Buckley\#, P.L. Hink\# \\ * Dept. of Physics 83 Astronomy, Louisiana State Univ., Baton Rouge, LA 70803

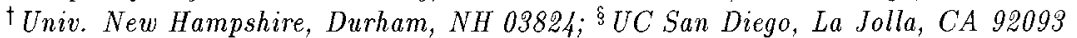 \\ \| UC Riverside, Riverside, CA 92521; \# Washington Univ., St. Louis, MO 63120 \\ $\ddagger$ Southern Univ., Baton Rouge, LA 70813; ${ }^{\$}$ Los Alamos Natl. Lab., Los Alamos, NM 87545
}

\begin{abstract}
MARGIE (Minute-of-Arc Resolution Gamma-ray Imaging Experiment) is a large area $\left(\sim 10^{4} \mathrm{~cm}^{2}\right)$, wide field-of-view $(\sim 1 \mathrm{sr})$, hard X-ray/gamma-ray $(\sim 20-$ $600 \mathrm{keV}$ ) coded-mask imaging telescope capable of performing a sensitive survey of both steady and transient cosmic sources. MARGIE has been selected for a NASA mission-concept study for an Ultra Long Duration (100 day) Balloon flight. We describe our program to develop the instrument based on new detector technology of either cadmium zinc telluride (CZT) semiconductors or pixellated cesium iodide (CsI) scintillators viewed by fast-timing bi-directional charge-coupled devices (CCDs). The primary scientific objectives are to image faint Gamma-Ray Bursts (GRBs) in nearreal-time at the low intensity (high-redshift) end of the $\log \mathrm{N}-\operatorname{logS}$ distribution, thereby extending the sensitivity of present observations, and to perform a wide field survey of the Galactic plane.
\end{abstract}

\section{INTRODUCTION}

Gamma-Ray Bursts (GRBs) are intense bursts of $\gamma$ radiation, lasting from fractions of a second to minutes, which emit the bulk of their energy above $0.1 \mathrm{MeV}$ (see e.g., Fishman and Meegan 1995; Band 1998). The origin and emission mechanism of GRBs are still quite uncertain. It has been long recognized that the key to unraveling the GRB mystery is the identification of burst counterparts at other wavelengths. Over the past two years, the BeppoSAX X-ray mission has localized over a dozen bursts to sufficiently small spatial regions (a few arc-minutes), on short enough timescales (a few hours) so that X-ray, optical and radio telescopes have detected the fading GRB afterglows. The recent multiwavelength observations which now include over four redshift measurements (Kulkarni et al. 1999) suggest that GRBs are cosmological in origin. 
A relativistic fireball model (see e.g., Piran 1998; Sari et al. 1998) has been reasonably successful in explaining the observed X-ray and optical afterglows. The $\gamma$-ray burst itself appears to be the result of internal shocks in the relativistic expanding ejecta and the forward shock moving through the interstellar medium produces the afterglows. Multiwavelength observations triggered by rapid GRB localization and notification provide a wealth of information about the physical conditions in the GRB environment. The richness and complexity of the fireball phenomenon and the observational differences between GRB events require a large statistical sample of observed bursts in order to fully unravel the physics of these sources.

The most recent report of NASA's Gamma Ray Working Group outlined a plan for the next years that included a hard X-ray $(<200 \mathrm{keV})$ survey as a high priority item. The basis for promoting a hard X-ray instrument was that the most recent survey was conducted with the HEAO-1 mission, more than 20 years ago. Since then most of the information we have collected about the hard X-ray sky comes from balloon instruments on day-long flights, from occultation monitoring of strong point sources with BATSE on GCRO, from investigations of point sources with the OSSE instrument on CGRO, and from partial sky surveys with moderate spatial resolution $\left(\sim 13^{\prime}\right)$ by the SIGMA instrument on GRANAT.

MARGIE (Minute-of-Arc Resolution Gamma-ray Imaging Experiment) is a large area $\left(\sim 10^{4} \mathrm{~cm}^{2}\right)$, wide field-of-view $(\sim 1 \mathrm{sr})$, hard X-ray/ $\gamma$-ray $(\sim 20-600 \mathrm{keV})$ coded-mask imaging telescope capable of performing a sensitive survey of both steady and transient cosmic sources. MARGIE has been selected for a NASA mission-concept study for an Ultra Long Duration (100 day) Balloon flight. The instrument is designed to observe 30-40 GRBs in a 100 day balloon flight with sufficient $\mathrm{S} / \mathrm{N}$ to permit $\mathrm{GRB}$ localizations to within $\sim 2$ arc-minutes. MARGIE is also ideal to conduct a hard X-ray mapping of the diffuse emission from the Galactic plane and to survey the hard X-ray sky.

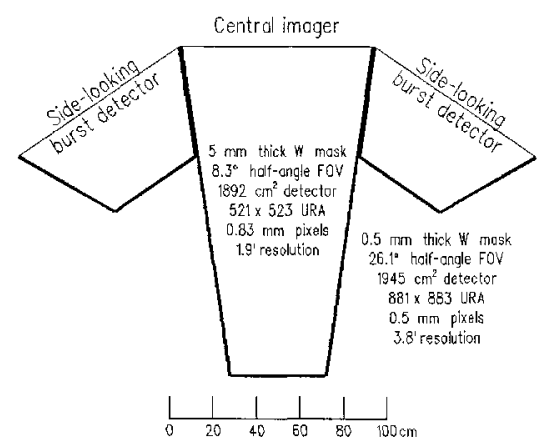

FIGURE 1. Schematic of the MARGIE $\gamma$-ray telescope. 


\section{MARGIE INSTRUMENT CONCEPT}

The essential components of the MARGIE experiment (Cherry et al. 1999; Stacy et al. 1999; McConnell et al. 1996) are the coded aperture mask and the CsI/CCD or CZT central detector. As shown in Figure 1, the instrument consists of five separate telescopes. The central telescope has an $87 \times 87 \mathrm{~cm}^{2} \times 5 \mathrm{~mm}$ thick tungsten mask viewed by an $1892 \mathrm{~cm}^{2} \mathrm{CsI} / \mathrm{CCD}$ array with $(0.8 \mathrm{~mm})^{2}$ pixels at a maskdetector separation of $150 \mathrm{~cm}$. The central telescope is designed for high resolution $\left(1.9^{\prime}\right)$, narrow $\left(8.3^{\circ}\right.$ half angle) field-of-view (FOV) measurements of point sources in addition to GRBs. The four side telescopes, each with $88 \times 88 \mathrm{~cm}^{2} \times 0.5 \mathrm{~mm}$ thick masks, a $1945 \mathrm{~cm}^{2}$ detector array, $0.5 \mathrm{~mm}$ pixels, and $45 \mathrm{~cm}$ mask-detector separation, provide excellent sky coverage and sensitivity $\left(26.1^{\circ}\right.$ half angle FOV, each with $3.8^{\prime}$ resolution) for GRBs and point sources. Plastic scintillators covering the masks provide an anticoincidence veto for charged cosmic rays interactions in the detector.

The coded aperture (or "multi-pinhole") technique works by allowing an absorbing mask to cast a shadow pattern on a position-sensitive detection plane (e.g., Caroli et al. 1987; Skinner et al. 1987). With a proper choice of mask pattern to minimize artifacts from the imaging process, the encoded pattern can then be processed to reproduce an image of the sky. The mask element geometry is defined by the mask thickness and the mask element size (or width). The mask thickness must be sufficient to attenuate photons (hence, modulate the incident flux) in the desired energy range. On the other hand, the thickness of the mask must be limited so as to maintain uniformity of mask transmission for off-axis sources. The telescope angular resolution corresponds to the angular size of a mask element as seen from the detection plane, and so is dictated by the mask element size and the mask-detector separation. Therefore the detector must be able to resolve the individual mask elements in the projected pattern; i.e., it must be able to locate events with an accuracy no larger than the mask element size. Any technology which improves the detector plane spatial resolution can therefore lead to an improvement in telescope angular resolution and sensitivity.

\section{DETECTOR PLANE TECHNOLOGIES}

The key enabling technology for MARGIE is the central detector. In order to obtain the fine-grained position resolution required in the plane of the central detector, two alternate position-sensitive $\gamma$-ray detector technologies are under development for use in MARGIE. The central detector will consist of five two-dimensional arrays of either $0.3 \mathrm{~mm}$ pitch cadmium zinc telluride (CZT) strip detectors or $0.5-0.9$ $\mathrm{mm}$ pitch segmented cesium iodide (CsI) scintillator. The CsI scintillators will be viewed by a Bi-Directional Charge Coupled Device (CCD) array designed for low-noise spectroscopy and fast timing $(10 \mu \mathrm{s})$ applications. 


\section{CZT Detectors}

Prototype cross-strip and planar CZT detectors have been flown and flight tested. The measured background in flight is low compared to the atmospheric flux entering the telescope aperture. The latest results on the overall CZT performance and background levels at balloon altitudes are presented by Slavis et al. in these proceedings. CZT detectors with orthogonal coplanar anode strips have also been developed. Sub-millimeter position resolutions and excellent energy resolution have been demonstrated. These results are presented by Ryan et al. elsewhere in these proceedings.

\section{Segmented-CsI arrays coupled to Bi-Directional CCDs}

Segmented CsI: CsI(Tl) is an efficient X-ray $/ \gamma$-ray scintillator due to its high density $\left(\sim 4.51 \mathrm{gm} / \mathrm{cm}^{3}\right)$ and large Z. Since the light is emitted essentially isotropically, the spot size diameter is comparable to the detector thickness. Therefore, one has competing requirements: a thicker detector for higher detection efficiency and a thinner detector for better position resolution. For MARGIE, the position resolution requirements demand a spot size $<0.5 \mathrm{~mm}$.

Pixellated CsI arrays offer a possible solution. Large area $\left(50 \times 50 \mathrm{~cm}^{2}\right)$ submillimeter pixel arrays up to $4 \mathrm{~cm}$ thick are commercially available (Krus et al. 1999 ) in several different scintillator materials (e.g., CsI, BGO, $\mathrm{CdWO}_{4}$ ) and produced with white paint, white epoxy or metal reflectors between the pixels. The pixel sizes are dictated by the mechanical properties of the crystal (e.g., hardness, cleavage plane). Evaluation of these arrays is currently underway.

Bi-Directional CCD: The segmented CsI scintillator output will be detected with a CCD. Standard CCDs are integrating devices operating at video rates (typically $\sim 30 \mathrm{~Hz}$ ). A balloon-borne $\gamma$-ray telescope, however, demands a faster time

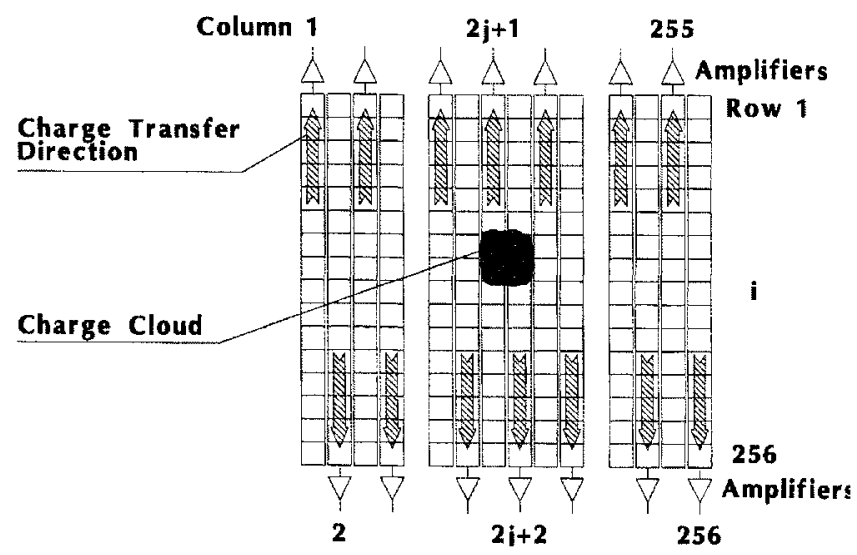

FIGURE 2. Schematic view of the Bi-Directional CCD. 
resolution to veto cosmic rays hitting the detector $(\sim 10 \mathrm{kHz})$ and isolate individual photons to measure their energies. We have therefore developed a Fast Timing Bi-Directional CCD with $10 \mu \mathrm{s}$ timing resolution and $50 \mu \mathrm{m}$ position resolution (Cherry et al. 1996).

The Bi-Directional CCD employs a continuous readout scheme in which charge collected in alternate pixel columns is clocked separately to the top and bottom of the CCD chip where it is amplified and read out (see Figure 2). The basic operation and performance characteristics of this device have been demonstrated with a set of prototype Bi-Directional CCDs fabricated for us at the Orbit Semiconductors foundry, based on a design by Suni Imaging Microsystems, Inc. (Cherry et al. 1999; Stacy et al. 1999). We are currently implementing the data readout circuitry for the Bi-Directional CCD in a $1.2 \mu \mathrm{m}$ CMOS ASIC design. Ultimately, both the CCD and readout electronics will be incorporated into a single monolithic CMOS chip. These further improvements will lead to an imaging device with exceptional lownoise performance and fast timing ideally suited for our astrophysical objectives.

\section{SUMMARY}

The MARGIE instrument will be a large-area, wide field-of-view, hard X-ray $/ \gamma$ ray imaging telescope. It will be capable of providing accurate positions, and of characterizing the temporal and spectral behavior of faint transient sources (e.g., GRBs) in near-real-time, for rapid counterpart searches. It will also carry out sensitive surveys for both steady and transient cosmic sources over the course of a 100-day Ultra Long Duration Balloon flight.

\section{REFERENCES}

1. G.J. Fishman and C.A. Meegan, Ann. Rev. Astron. Astrophys. 33, 415 (1995)

2. D. Band, in $8^{\text {th }}$ Marcel Grossmann Mtg. on General Relativity, ed. T. Piran, in press (astro-ph/9712193, 1998)

3. S. R. Kulkarni et al., Nature, submitted (astro-ph/9902272 v2, 1999).

4. T. Piran, preprint, astro-ph/9810256 (1998)

5. R. Sari, T. Piran and R. Narayan, ApJ 497 L17 (1998)

6. M.L. Cherry et al., Proc. SPIE 3765, in press (1999)

7. J.G. Stacy et al., Proc. $19^{\text {th }}$ Texas Symposium (Paris), in press (1999)

8. M. McConnell et al., Proc. SPIE 2806, 349 (1996)

9. E. Caroli et al., Space Sci. Rev. 45, 349 (1987)

10. G.K. Skinner et al., Astrophys.Space Sci. 136, 337 (1987)

11. K. Slavis et al., these proceedings

12. J. Ryan et al., these proceedings

13. D. Krus et al., Proc. SPIE Conf. 3768, in press (1996)

14. M.L. Cherry et al., Proc. SPIE 2806, 551 (1996) 